\author{
A. M. Kytmanov, E. K. Myshinina
}

\title{
ON FINDING THE RESULTANT OF TWO ENTIRE FUNCTIONS
}

\begin{abstract}
Using Newton's recurrent formulae, we find the product of values of an entire function of one variable in zeroes of another entire function. This allows to answer whether they have common zeros. By that, we propose an approach to construction of the resultant of two entire functions. We also give examples illustrating the main result.
\end{abstract}

Key words: resultant, entire function, Newton formulae

2010 Mathematical Subject Classification: 13P15, 32A15

1. Introduction. The classical resultant $R(f, g)$ of two polynomials $f$ and $g$ may be defined variously: using the Sylvester determinant (see e.g. [1], [10], [16]), the product formula $R(f, g)=\prod_{\{x: f(x)=0\}} g(x)$ (see [1], [10], [16]), or the Bezout-Caley approach (see [9]).

In this paper, we give a constructive approach to define a resultant of two entire functions of one complex variable using the product formula. Our choice is justified by the fact that entire functions are the direct generalization of polynomials.

In a series of papers (see [4-6], [8], [15]) various authors proposed generalizations for the resultant of analytic functions in the ring of matrixvalued functions, meromorphic functions on Riemann surfaces, for systems of algebraic and transcendent equations. In all these investigations, it is assumed that the number of zeroes and poles is finite. In our case, functions may have infinite number of zeroes but instead we need to employ the limit procedure.

The interest to this problem is explained by the fact that many mathematical models require studying non-algebraic equations and systems, for example, equations of chemical kinetics often use exponential polynomials [2].

(c) Petrozavodsk State University, 2020 
The first step in defining the resultant of two entire functions was done in [11], where authors studied the case of an entire function and a polynomial (or an entire function with a finite number of zeroes). The conditions for an entire function to have a finite number of zeroes were studied in [12]. The paper [13] generalizes the results of [11] to the case when one of entire functions satisfies some strict conditions, but may have infinite number of zeroes.

Our approach allows to determine if entire functions have common zeroes without computing the zeroes. Our formulae for the resultant involve power sums of roots, which may be found by Newton's formulae without finding the zeroes.

2. A cubic polynomial and an entire function. To begin with, we consider a cubic polynomial $f(z)$ and a polynomial $g(z)$ of degree $n$ :

$$
\left\{\begin{array}{l}
f(z)=a_{0}+a_{1} z+a_{2} z^{2}+z^{3} \\
g(z)=b_{0}+b_{1} z+b_{2} z^{2}+\ldots+b_{n} z^{n} .
\end{array}\right.
$$

Denote the roots of $f(z)$ by $z_{1}, z_{2}, z_{3}$. Then the resultant $R(f, g)$ of $f$ and $g$ is computed as

$$
\begin{array}{r}
R(f, g)=\prod_{i=1}^{3} g\left(z_{i}\right)=g\left(z_{1}\right) \cdot g\left(z_{2}\right) \cdot g\left(z_{3}\right)=\sum_{k=0}^{n} b_{k}^{3}\left(z_{1} z_{2} z_{3}\right)^{k}+ \\
+\sum_{s=0}^{n} \sum_{t=s+1}^{n}\left(z_{1} z_{2} z_{3}\right)^{s}\left(b_{s} b_{t}^{2}\left[\left(z_{1} z_{2}\right)^{t-s}+\left(z_{1} z_{3}\right)^{t-s}+\left(z_{2} z_{3}\right)^{t-s}\right]+\right. \\
\left.+b_{s}^{2} b_{t}\left[z_{1}^{t-s}+z_{2}^{t-s}+z_{3}^{t-s}\right]\right)+ \\
+\sum_{s=0}^{n} \sum_{t=s+1}^{n} \sum_{p=t+1}^{n} b_{s} b_{t} b_{p}\left(z_{1} z_{2} z_{3}\right)^{s}\left[z_{1}^{p-s} z_{2}^{t-s}+z_{2}^{p-s} z_{1}^{t-s}+z_{1}^{p-s} z_{3}^{t-s}+\right. \\
\left.\quad+z_{3}^{p-s} z_{1}^{t-s}+z_{2}^{p-s} z_{3}^{t-s}+z_{3}^{p-s} z_{2}^{t-s}\right] .
\end{array}
$$

Note that the expressions in $z_{j}$ 's in these sums are symmetric polynomials. This means that they can be expressed via the coefficients of $f(z)$, however, we rewrite them using the power sums of the roots:

$$
S_{k}=z_{1}^{k}+z_{2}^{k}+z_{3}^{k}, \quad k \in \mathbb{N} .
$$

If necessary, $S_{k}$ 's may be rewritten via the elementary symmetric poly- 
nomials $e_{j}$ 's of the roots:

$$
\left\{\begin{array}{l}
e_{1}=z_{1}+z_{2}+z_{3} \\
e_{2}=z_{1} \cdot z_{2}+z_{1} \cdot z_{3}+z_{2} \cdot z_{3} \\
e_{3}=z_{1} \cdot z_{2} \cdot z_{3}
\end{array}\right.
$$

using the Newton-Girard formula (see, e.g., [3], [7, formula 8]):

$$
S_{k}=\sum_{\substack{r_{1}+2 r_{2}+\ldots+k r_{k}=k, r_{1}, r_{2}, \ldots, r_{k} \geqslant 0}}(-1)^{k} \frac{k\left(r_{1}+\ldots+r_{k}-1\right) !}{r_{1} ! \cdot \ldots \cdot r_{k} !} \prod_{i=1}^{k}\left(-e_{i}\right)^{r_{i}}
$$

Viete's formulae allow to rewrite these in terms of the coefficients of $f(z)$. It is easy to see that

$$
z_{1}^{k} z_{2}^{l}+z_{1}^{l} z_{2}^{k}+z_{1}^{k} z_{3}^{l}+z_{1}^{l} z_{3}^{k}+z_{2}^{k} z_{3}^{l}+z_{2}^{l} z_{3}^{k}=S_{k} \cdot S_{l}-S_{k+l}, \quad k, l \in \mathbb{N} .
$$

and

$$
\left(z_{1} z_{2}\right)^{k}+\left(z_{1} z_{3}\right)^{k}+\left(z_{2} z_{3}\right)^{k}=\frac{1}{2}\left(S_{k}^{2}-S_{2 k}\right) .
$$

Therefore, the expression (2) becomes

$$
\begin{aligned}
R=\prod_{i=1}^{3} g\left(z_{i}\right)= & \sum_{k=0}^{n} b_{k}^{3}\left(-a_{0}\right)^{k}+ \\
& +\sum_{s=0}^{n} \sum_{t=s+1}^{n}\left(-a_{0}\right)^{s}\left(\frac{1}{2} b_{s} b_{t}^{2}\left(S_{t-s}^{2}-S_{2 t-2 s}\right)+b_{s}^{2} b_{t} S_{t-s}\right)+ \\
& +\sum_{s=0}^{n} \sum_{t=s+1}^{n} \sum_{p=t+1}^{n} b_{s} b_{t} b_{p}\left(-a_{0}\right)^{s}\left[S_{t-s} \cdot S_{p-s}-S_{t+p-2 s}\right] .
\end{aligned}
$$

Thus, we arrive at the first result:

Theorem 1. The resultant $R(f, g)$ of the polynomials (1) is given by (5).

Passing to the limit as $n \rightarrow \infty$ in (5), we get the result for a system consisting of a cubic polynomial and an entire function.

Theorem 2. Let $g(z)$ be an entire function in one complex variable and

$$
g(z)=b_{0}+b_{1} z+b_{2} z^{2}+\ldots+b_{n} z^{n}+\ldots,
$$


be its series expansion, and let $f(z)$ be a cubic polynomial as in (1). Then the resultant $R(f, g)$ is given by

$$
\begin{aligned}
R(f, g) & =\sum_{k=0}^{\infty} b_{k}^{3}\left(-a_{0}\right)^{k}+ \\
& +\sum_{s=0}^{\infty} \sum_{t=s+1}^{\infty}\left(-a_{0}\right)^{s}\left(\frac{1}{2} b_{s} b_{t}^{2}\left(S_{t-s}^{2}-S_{2 t-2 s}\right)+b_{s}^{2} b_{t} S_{t-s}\right)+ \\
& +\sum_{s=0}^{\infty} \sum_{t=s+1}^{\infty} \sum_{p=t+1}^{\infty} b_{s} b_{t} b_{p}\left(-a_{0}\right)^{s}\left[S_{t-s} \cdot S_{p-s}-S_{t+p-2 s}\right]
\end{aligned}
$$

provided the series in the right-hand side converges absolutely.

3. A case of two polynomials. Consider a system of two equations consisting of two polynomials of degrees $m$ and $n$

$$
\left\{\begin{array}{l}
f(z)=a_{0}+a_{1} z+a_{2} z^{2}+a_{3} z^{3}+\ldots+a_{m-1} z^{m-1}+z^{m} \\
g(z)=b_{0}+b_{1} z+b_{2} z^{2}+\ldots+b_{n} z^{n} .
\end{array}\right.
$$

The case $m=2$ has been considered in [14], and the case $m=3$ has been considered above.

Denote the roots of $f(z)$ by $z_{1}, z_{2}, \ldots, z_{m}$ (there may be multiple roots among them). And $S_{k}$ are there power sums of order $k$. Elementary but cumbersome calculations show that

$$
\prod_{i=1}^{m} g\left(z_{i}\right)=G_{1}+G_{2}+\ldots+G_{i}+\ldots+G_{m},
$$

where each $G_{i}$ consists of several multiple sums. Namely,

$$
\begin{aligned}
G_{1} & =\sum_{s=0}^{n}(-1)^{m s} a_{0}^{s} b_{s}^{m} \\
G_{2} & =\sum_{s=0}^{n} \sum_{t=s+1}^{n}(-1)^{m s} a_{0}^{s} b_{s}^{m-1} b_{t} S_{t-s}+ \\
& +\sum_{s=0}^{n} \sum_{t=s+1}^{n}(-1)^{m s} a_{0}^{s} b_{s}^{m-2} b_{t}^{2}\left(\frac{S_{t-s}^{2}-S_{2 t-2 s}}{2}\right)+ \\
& +\sum_{s=0}^{n} \sum_{t=s+1}^{n}(-1)^{m s} a_{0}^{s} b_{s}^{m-3} b_{t}^{3}\left(\frac{S_{t-s}^{3}-3 S_{2 t-2 s} \cdot S_{t-s}+2 S_{3 t-3 s}}{4}\right)+
\end{aligned}
$$




$$
\begin{aligned}
& +\sum_{l=4}^{m-1} \sum_{s=0}^{n} \sum_{t=s+1}^{n}(-1)^{m s} a_{0}^{s} b_{s}^{m-l} b_{t}^{l}\left(\frac{S_{t-s}^{l}-l S_{t-s} \cdot S_{(l-1)(t-s)}+(l-1) S_{l(t-s)}}{2^{l-1}}\right), \\
& G_{3}=\sum_{s=0}^{n} \sum_{t=s+1}^{n} \sum_{p=t+1}^{n}(-1)^{m s} a_{0}^{s} b_{s}^{m-2} b_{t} b_{p}\left(S_{t-s} \cdot S_{p-s}-S_{t+p-2 s}\right)+ \\
& +\sum_{s=0}^{n} \sum_{t=s+1}^{n} \sum_{p=t+1}^{n}(-1)^{m s} a_{0}^{s} b_{s}^{m-3} b_{t}^{2} b_{p} \times \\
& \times\left(\frac{S_{t-s}^{2} \cdot S_{p-s}-S_{2 t-2 s} \cdot S_{p-s}-2 S_{t+p-2 s} \cdot S_{t-s}+2 S_{2 t+p-3 s}}{2}\right)+ \\
& +\sum_{s=0}^{n} \sum_{t=s+1}^{n} \sum_{p=t+1}^{n}(-1)^{m s} a_{0}^{s} b_{s}^{m-3} b_{t} b_{p}^{2} \times \\
& \times\left(\frac{S_{p-s}^{2} \cdot S_{t-s}-S_{2 p-2 s} \cdot S_{t-s}-2 S_{t+p-2 s} \cdot S_{p-s}+2 S_{2 p+t-3 s}}{2}\right)+ \\
& +\sum_{l=4}^{m-1} \sum_{\substack{\beta_{1}+\beta_{2}=l \\
\beta_{1} \geqslant 1, \beta_{2} \geqslant 1}} \sum_{s=0}^{n} \sum_{t=s+1}^{n} \sum_{p=t+1}^{n}(-1)^{m s} a_{0}^{s} b_{s}^{m-l} b_{t}^{\beta_{1}} b_{p}^{\beta_{2}} \times \\
& \times\left(\frac{S_{p-s}^{\beta_{2}} \cdot S_{t-s}^{\beta_{1}}-\beta_{2} S_{p-s} \cdot S_{\beta_{1}(t-s)+(p-s)\left(\beta_{2}-1\right)}-}{2^{l-2}}\right. \\
& \left.\underline{-\beta_{1} S_{t-s} \cdot S_{\beta_{2}(p-s)+(t-s)\left(\beta_{1}-1\right)}+\left(\beta_{1}+\beta_{2}-1\right) S_{\beta_{1}(t-s)+\beta_{2}(p-s)}}\right), \\
& G_{4}=\sum_{s=0}^{n} \sum_{t=s+1}^{n} \sum_{p=t+1}^{n} \sum_{r=p+1}^{n}(-1)^{m s} a_{0}^{s} b_{s}^{m-3} b_{t} b_{p} b_{r} \times \\
& \times\left(S_{t-s} \cdot S_{p-s} \cdot S_{r-s}-S_{t+p-2 s} \cdot S_{r-s}-S_{t+r-2 s} \cdot S_{p-s}-\right. \\
& \left.-S_{p+r-2 s} \cdot S_{t-s}+2 S_{p+t+r-3 s}\right)+ \\
& +\sum_{l=4}^{m-1} \sum_{\substack{\beta_{1}+\beta_{2}+\beta_{3}=l \\
\beta_{1} \geqslant 1, \beta_{2} \geqslant 1, \beta_{3} \geqslant 1}} \sum_{s=0}^{n} \sum_{t=s+1}^{n} \sum_{p=t+1}^{n} \sum_{r=p+1}^{n}(-1)^{m s} a_{0}^{s} b_{s}^{m-l} b_{t}^{\beta_{1}} b_{p}^{\beta_{2}} b_{r}^{\beta_{3}} \times \\
& \times\left(\frac{S_{t-s}^{\beta_{1}} \cdot S_{p-s}^{\beta_{2}} \cdot S_{r-s}^{\beta_{3}}-\beta_{1} S_{t-s} \cdot S_{\beta_{2}(p-s)+\beta_{3}(r-s)+\left(\beta_{1}-1\right)(t-s)}-}{2^{l-3}}\right.
\end{aligned}
$$




$$
\begin{aligned}
& -\beta_{2} S_{p-s} \cdot S_{\beta_{1}(t-s)+\beta_{3}(r-s)+\left(\beta_{2}-1\right)(p-s)}-\beta_{3} S_{r-s} \cdot S_{\beta_{1}(t-s)+\beta_{2}(p-s)+\left(\beta_{3}-1\right)(r-s)}+ \\
& \left.+\left(\beta_{1}+\beta_{2}+\beta_{3}-1\right) S_{\beta_{1}(t-s)+\beta_{2}(p-s)+\beta_{3}(r-s)}\right) .
\end{aligned}
$$

If we denote the summation indices by $\alpha_{1}, \alpha_{2}, \ldots, \alpha_{m}$ and differences of indices by $j_{2}=\alpha_{2}-\alpha_{1}, j_{3}=\alpha_{3}-\alpha_{1}, \ldots, j_{m}=\alpha_{m}-\alpha_{1}$, then

$$
\begin{aligned}
G_{m} & =\sum_{\alpha_{1}=0}^{n} \sum_{\alpha_{2}=\alpha_{1}+1}^{n} \sum_{\alpha_{3}=\alpha_{2}+1}^{n} \ldots \sum_{\alpha_{m}=\alpha_{m-1}+1}^{n}(-1)^{m \alpha_{1}} a_{0}^{\alpha_{1}} b_{\alpha_{1}}^{m-(m-1)} b_{\alpha_{2}} \ldots b_{\alpha_{m}} \times \\
& \times\left(\prod_{k=2}^{m} S_{j_{k}}-\sum_{k=2}^{m} S_{j_{k}} \cdot S_{j_{2}+\ldots+j_{k-1}+j_{k+1}+\ldots+j_{m}}+(m-2) S_{j_{2}+j_{3}+\ldots+j_{m}}\right) .
\end{aligned}
$$

Remark. If $f(z)$ is a polynomial of degree $m$, then $G_{m}$ consists of one m-tuple sum.

Theorem 3. The resultant $R(f, g)$ of the system of polynomials (6) is given by (7).

The proof follows easily by induction over $m$ with the base for $m=3$.

4. A general case. For the general case, it is enough to pass to the limit as $n$ and $m$ tend to infinity.

Theorem 4. Let $f(z)$ and $g(z)$ be entire functions of one complex variable

$$
\begin{aligned}
& f(z)=a_{0}+a_{1} z+a_{2} z^{2}+\ldots+a_{m} z^{m}+\ldots, \\
& g(z)=b_{0}+b_{1} z+b_{2} z^{2}+\ldots+b_{n} z^{n}+\ldots
\end{aligned}
$$

Then the resultant $R(f, g)$ is given by formula (7), where we pass to the limit as $n$ and $m$ tend to infinity. The function $f(z)$ must be transformed to the form (6) by dividing it on $a_{m}$.

We suggest also another approach to obtain these formulae, which is more convenient for algorithmic realization. Let

$$
\begin{aligned}
& f(z)=a_{0}+a_{1} z+a_{2} z^{2}+\ldots+z^{m} \\
& g(z)=b_{0}+b_{1} z+b_{2} z^{2}+\ldots+b_{n} z^{n}+\ldots
\end{aligned}
$$


Denote the roots of $f(z)$ by $z_{1}, \ldots, z_{m}$. Then the resultant $R(f, g)$ of $f$ and $g$ is

$$
R=\prod_{i=1}^{m} g\left(z_{i}\right)=\left(\sum_{j_{1}=0}^{\infty} b_{j_{1}} z_{1}^{j_{1}}\right) \ldots\left(\sum_{j_{m}=0}^{\infty} b_{j_{m}} z_{m}^{j_{m}}\right) .
$$

Each term in this product is in one-to-one correspondence with a point of $\mathbb{Z}_{\geqslant}^{n}$. To group all the terms into symmetric polynomials, we subdivide $\mathbb{Z}_{\geqslant}^{n}$ into cones. In fact, due to symmetry, it is enough to describe only one cone containing exactly one monomial from each symmetric polynomial in (8). For this, we denote the standard base vectors of $\mathbb{Z}^{n}$ by $e_{1}, \ldots, e_{n}$ and define vectors

$$
v_{k}=\sum_{j=1}^{k} e_{j}, \quad j=1, \ldots, n .
$$

These vectors generate the required simplicial cone. The faces of this cone always intersect; to avoid this, we consider the following sets of integer points lying in partially closed cones:

$$
\begin{aligned}
\sigma_{0} & =\mathbb{Z}_{\geqslant} v_{n} ; \\
\sigma_{1}^{k} & =\mathbb{Z}_{\geqslant} v_{n}+\mathbb{Z}_{>} v_{k}, \quad k=1, \ldots, n-1 ; \\
\sigma_{2}^{\mathbf{k}} & =\mathbb{Z}_{\geqslant} v_{n}+\mathbb{Z}_{>} v_{k_{1}}+\mathbb{Z}_{>} v_{k_{2}}, \mathbf{k}=\left(k_{1}, k_{2}\right), k_{1}, k_{2}=1, \ldots, n-1, k_{1} \neq k_{2} ; \\
& \cdots \\
\sigma_{n} & =\mathbb{Z}_{\geqslant} v_{n}+\mathbb{Z}_{>} v_{1}+\cdots+\mathbb{Z}_{>} v_{n-1} .
\end{aligned}
$$

A point $\xi$ of any of these sets encodes a monomial

$$
b_{\xi} z^{\xi}=b_{\xi_{1}} \ldots b_{\xi_{n}} z_{1}^{\xi_{1}} \ldots z_{n}^{\xi_{n}}
$$

Each set $\sigma$ from the list above produces the series

$$
\sum_{\alpha \in \sigma} b_{\alpha} \operatorname{Sym}\left(z^{\alpha}\right)
$$

where $\operatorname{Sym}\left(z^{\alpha}\right)$ is the sum of elements of the orbit of $z^{\alpha}$ under the action of the symmetric group

$$
\operatorname{Sym}\left(z^{\alpha}\right)=\sum_{p \text { is a permutation }} z^{p(\alpha)}
$$


For example, $\sigma_{0}$ gives the series

$$
\sum_{s=0}^{\infty} b_{s \cdot v_{n}} \operatorname{Sym}\left(z^{s \cdot v_{n}}\right)=\sum_{s=0}^{\infty} b_{s}^{n}\left(z_{1} \ldots z_{n}\right)^{s}
$$

and $\sigma_{1}^{1}$

$$
\begin{gathered}
\sum_{s=0}^{\infty} \sum_{t=1}^{\infty} b_{s \cdot v_{n}+t \cdot v_{1}} \operatorname{Sym}\left(z^{s \cdot v_{n}+t \cdot v_{1}}\right)= \\
=\sum_{s=0}^{\infty} \sum_{t=1}^{\infty} b_{s+t} b_{s}^{n-1}\left(z_{1}^{s+t} z_{2}^{s} \ldots z_{n}^{s}+\cdots+z_{1}^{s} z_{2}^{s} \ldots z_{n}^{s+t}\right)= \\
=\sum_{s=0}^{\infty} \sum_{t=1}^{\infty} b_{s+t} b_{s}^{n-1}\left(z_{1} \ldots z_{n}\right)^{s}\left(z_{1}^{t}+\ldots+z_{n}^{t}\right)
\end{gathered}
$$

The symmetric polynomials in each series (9) can be expressed via coefficients of $f$ and power sums of its roots by standard procedures of computer algebra systems or by formulae from [3]. Summing up over all $\sigma$ from the list and passing to the limit as $m$ tends to infinity, we obtain the result of Theorem 4, provided that all the series converge absolutely.

5. Examples. In this section, we consider examples that demonstrate the main results of the paper, as well as examples leading to computation of some earlier unknown sums of multiple series. These results are of independent interest. We begin with a simple example illustrating Theorem 1, where we compute all the quantities in formula (5).

Example 1. Consider the system of equations $(n=2)$

$$
\left\{\begin{array}{l}
f(z)=z(z-1)(z+1)=z^{3}-z \\
g(z)=(z-2)(z+2)=z^{2}-4
\end{array}\right.
$$

In this case,

$$
a_{0}=0, \quad a_{1}=-1, \quad a_{2}=0, \quad b_{0}=-4, \quad b_{1}=0, \quad b_{2}=1 .
$$

Then formula (5) takes the form

$$
R(f, g)=\sum_{k=0}^{2} b_{k}^{3}\left(-a_{0}\right)^{k}+\sum_{s=0}^{2} \sum_{t=s+1}^{2}\left(-a_{0}\right)^{s}\left(\frac{1}{2} b_{s} b_{t}^{2}\left(S_{t-s}^{2}-S_{2 t-2 s}\right)+b_{s}^{2} b_{t} S_{t-s}\right)+
$$




$$
+\sum_{s=0}^{2} \sum_{t=s+1}^{2} \sum_{p=t+1}^{2} b_{s} b_{t} b_{p}\left(-a_{0}\right)^{s}\left[S_{t-s} \cdot S_{p-s}-S_{t+p-2 s}\right] .
$$

Since $a_{0}=0$, we get only terms that correspond to $k=0$ and $s=0$ in these sums, that are:

$$
\begin{aligned}
R(f, g)=b_{0}^{3}+\sum_{t=1}^{2}\left(\frac{1}{2} b_{0} b_{t}^{2}\left(S_{t}^{2}-S_{2 t}\right)\right. & \left.+b_{0}^{2} b_{t} S_{t}\right)+ \\
& +\sum_{t=1}^{2} \sum_{p=t+1}^{2} b_{0} b_{t} b_{p}\left[S_{t} \cdot S_{p}-S_{t+p}\right] .
\end{aligned}
$$

Since $b_{1}=0$, the one-dimensional sum has only summands for $t=2$ and the double sum has no non-zero terms. Thus,

$$
R(f, g)=b_{0}^{3}+\frac{1}{2} b_{0} b_{2}^{2}\left(S_{2}^{2}-S_{4}\right)+b_{0}^{2} b_{2} S_{2} .
$$

We find the power sums of the roots $S_{2}$ and $S_{4}$ without using the roots themselves, but just formulae (3) and Viete's formulae. In our case

$$
S_{2}=2, \quad S_{4}=2 .
$$

Thus, $R(f, g)=-36$.

Example 2. Consider the system of equations

$$
\left\{\begin{array}{l}
f(z)=z^{3}-a^{3} \\
g(z)=b_{0}+b_{1} z+b_{2} z^{2}+\ldots+b_{n} z^{n} .
\end{array}\right.
$$

This example demonstrates Theorem 1. According to the notation introduced above,

$$
z_{1}=a, \quad z_{2,3}=\frac{-a \pm a \sqrt{3} i}{2}
$$

We have

$$
\left\{\begin{array}{l}
e_{3}=z_{1} z_{2} z_{3}=a^{3} \\
e_{2}=z_{1} z_{2}+z_{1} z_{3}+z_{2} z_{3}=0 \\
e_{1}=z_{1}+z_{2}+z_{3}=0
\end{array}\right.
$$

The expressions $S_{k}$ are distinct from zero only if $k$ is a multiple of 3 , and then they are equal to

$$
S_{k}=z_{1}^{k}+z_{2}^{k}+z_{3}^{k}=3 a^{k} .
$$


For such $k$, we get

$$
\left(z_{1} z_{2}\right)^{k}+\left(z_{1} z_{3}\right)^{k}+\left(z_{2} z_{3}\right)^{k}=\frac{1}{2}\left(S_{k}^{2}-S_{2 k}\right)=3 a^{2 k} .
$$

Thus, by (5) we have

$$
\begin{aligned}
\prod_{i=1}^{3} g\left(z_{i}\right)=\sum_{s=0}^{n} b_{s}^{3} a^{3 s} & +\sum_{s=0}^{n} \sum_{j=1}^{\left[\frac{n-s}{3}\right]} a^{3 s}\left(3 b_{s} b_{s+3 j}^{2} a^{6 j}+3 b_{s}^{2} b_{s+3 j} a^{3 j}\right)+ \\
& +\sum_{s=0}^{n} \sum_{t=s+1}^{n} \sum_{p=t+1}^{n} b_{s} b_{t} b_{p} a^{3 s}\left[S_{t-s} \cdot S_{p-s}-S_{t+p-2 s}\right] .
\end{aligned}
$$

In the last expression, the triple sum is distinct from zero only if either both lower indices are multiples of 3 , or they are not but their sum is.

Example 3. Consider the system

$$
\left\{\begin{array}{l}
f(z)=z^{3}-a^{3} \\
g(z)=e^{b z}=\sum_{n=1}^{\infty} \frac{(b z)^{n}}{n !}=1+b z+\frac{(b z)^{2}}{2 !}+\ldots+\frac{(b z)^{n}}{n !}+\ldots
\end{array}\right.
$$

Using Theorem 2, we get

$$
\begin{gathered}
\prod_{i=1}^{3} g\left(z_{i}\right)= \\
=\sum_{k=0}^{\infty} \frac{(a b)^{3 k}}{(k !)^{3}}+\sum_{s=0}^{\infty} \sum_{j=1}^{\infty} a^{3 s}\left(3 \frac{b^{3 s+6 j}}{s !(s+3 j) !^{2}} a^{6 j}+3 \frac{b^{3 s+3 j}}{s !^{2}(s+3 j) !} a^{3 j}\right)+ \\
+\sum_{s=0}^{\infty} \sum_{t=s+1}^{\infty} \sum_{p=t+1}^{\infty} b_{s} b_{t} b_{p} a^{3 s}\left[S_{t-s} \cdot S_{p-s}-S_{t+p-2 s}\right]=1 .
\end{gathered}
$$

Acknowledgment. The first author was supported by Krasnoyarsk Mathematical Center and financed by the Ministry of Science and Higher Education of the Russian Federation in the framework of the establishment and development of regional Centers for Mathematics Research and Education (Agreement No. 075-02-2020-1534/1), the second author was supported by the RFBR (grant 19-31-60012).

\section{References}

[1] Bourbaki N. Algebra. Polynomials and Fields, Ordered Groups. Nauka, Moscow, 1965 [Russian translation]. 
[2] Bykov V. I., Tsybenova S. B. Nonlinear Models of Chemical Kinetics. KRASAND, Moscow, 2011 (in Russian).

[3] Chamberlin S., Rafizadeh A. A generalized Newton-Girard formula for monomial symmetric polynomials. Rocky Mountain J. Math., 2020, vol. 50, no. 3, pp. 941-946. DOI: https://doi.org/10.1216/rmj.2020.50.941

[4] Gohberg I. C., Heinig G. Resultant Matrix and its Generalization. I. Resultant Operator of Matrix Polynomial. Acta Sci. Math., 1975, vol. 72, pp. $41-61$.

[5] Gohberg I. C., Heinig G. Resultant Matrix and its Generalization. II. Continual Analog of Resultant Matrix. Acta Math. Acad. Sci. Hungar, 1976, vol. 28, pp. $189-209$.

[6] Gohberg I. C., Lerer L. E. Resultant Operators of a Pair of Analytic Functions. Proc. Amer. Math. Soc., 1978, vol. 72, no. 1, pp. 65-73.

[7] Gould H. W. The Girard-Waring power sum formulas for symmetric functions and Fibonacci sequences. Fibonacci Quart., 1999, vol. 37, no. 2, pp. $135-140$.

[8] Gustafsson B., Tkachev V. G. The Resultant on Compact Riemann Surfaces. Comm. Math. Physics, 2009, vol. 10, pp. 265-308.

DOI: https://doi.org/DOI:10.1007/s00220-008-0622-2

[9] Krein M. G., Naimark M. A. The Method of Symmetric and Hermitian Forms in the Theory of the Separation of the Roots of Algebraic Equation. Linear and Multilinear Algebra, 1981, vol. 10, no. 4, pp. 265-308.

DOI: https://doi.org/10.1080/03081088108817420

[10] Kurosh A. G. Course in Higher Algebra. Nauka, Moscow, 1968 (Russian translation).

[11] Kytmanov A. M., Naprienko Ya. M. An Approach to Define the Resultant of Two Entire Functions. Complex variables and elliptic equat., 2017, vol. 62, no. 2 , pp. $269-286$.

DOI: https://doi.org/10.1080/17476933.2016.1218855

[12] Kytmanov A. M., Khodos O. V. On Localization of Zeros of an Entire Function of Finite Order of Growth. Complex analysis and operator theory, 2017, vol. 11, no. 2, pp. 393-416.

DOI: https://doi.org/10.1007/s11785-016-0606-8

[13] Kytmanov A. M., Khodos O. V. An Approach to the Determination of the Resultant of Two Entire Functions. Russian Mathematics, 2018, vol. 62, no. 4 , pp. $42-51$. DOI: https://doi.org/10.3103/S1066369X18040059

[14] Kytmanov A. M., Myshkina E. K. On Some Approach for Finding the Resultant of Two Entire Functions. J. Siberian Federal Univ. Math. Phys., 
2019, vol. 12 , no. 4 , pp. $434-438$.

DOI: https://doi.org/10.17516/1997-1397-2019-12-4-434-438

[15] Morozov A. Yu., Shakirov Sh. R. New and Old Results in Resultant Theory. Theor. math. phys., 2010, vol. 163, no. 2, pp. 587-617.

DOI: https://doi.org/10.1007/s11232-010-0044-0

[16] van der Waerden B. L. Algebra. Springer-Verlag, Berlin-Heidelderg-New York, 1966.

Received May 29, 2020.

In revised form, August 16, 2020.

Accepted August 16, 2020.

Published online September 3, 2020.

A. M. Kytmanov

Siberian Federal University,

660041, 79, Svobodny pr., Krasnoyarsk, Russia

E-mail: akytmanov@sfu-kras.ru

E. K. Myshkina

Institute of Computational Modelling, SB RAS

660036, 50/44, Akademgorodok, Krasnoyarsk, Russia

E-mail: elfifenok@mail.ru 